Bull. Korean Math. Soc. 52 (2015), No. 4, pp. 1139-1148

http://dx.doi.org/10.4134/BKMS.2015.52.4.1139

\title{
CYCLIC BRANCHED COVERS OF ALTERNATING KNOTS AND $L$-SPACES
}

\author{
Masakazu Teragaito
}

\begin{abstract}
For any alternating knot, it is known that the double branched cover of the 3 -sphere branched over the knot is an $L$-space. We show that the three-fold cyclic branched cover is also an $L$-space for any genus one alternating knot.
\end{abstract}

\section{Introduction}

An $L$-space $M$ is a rational homology 3 -sphere whose Heegaard Floer homology $\widehat{H F}(M)$ is a free abelian group of rank equal to $\left|H_{1}(M ; \mathbb{Z})\right|([12])$. Prototypical examples of $L$-spaces are lens spaces. In recent years, it is recognized that $L$-spaces form an important class of 3-manifolds. For example, see $[2,12]$.

We consider the problem when cyclic branched covers of the 3-sphere branched over a knot or link is an $L$-space. Toward this direction, Ozsváth and Szabó [13] first showed that the double branched cover of any non-split alternating link (more generally, quasi-alternating link) is an $L$-space. Peters [15] verified that for a genus one, 2-bridge knot $C[2 m, 2 n](m, n>0)$ in Conway's notation, the $d$-fold cyclic branched cover is an $L$-space for any $d \geq 2$, and that for $C[2 m,-2 n](m, n>0)$, so is the 3 -fold cyclic branched cover. For the latter, the same conclusion still holds for the cases $d=4([17])$ and $d=5([9])$, but it would be false for sufficiently large $d([10,18])$.

In this paper, we restrict ourselves to alternating knots. As mentioned above, the double branched cover of any alternating knot is an $L$-space. Then, is the 3 -fold cyclic branched cover an $L$-space? The answer is positive for genus one, 2 -bridge knots. However, it is negative, in general. Let $\Sigma_{d}(K)$ denote the $d$ fold cyclic branched cover of the 3 -sphere branched over a knot $K$. By Baldwin [1], if $K$ is the trefoil, then $\Sigma_{d}(K)$ is an $L$-space if and only if $d \leq 5$. This implies that if $K$ is a $(2, m)$-torus knot with $m \geq 7$, then $\Sigma_{3}(K)$ is not an

Received June 30, 2014.

2010 Mathematics Subject Classification. Primary 57M25.

Key words and phrases. branched cover, alternating knot, pretzel knot, $L$-space.

The author was partially supported by Japan Society for the Promotion of Science, Grantin-Aid for Scientific Research (C), 25400093. 
$L$-space. Indeed, $\Sigma_{3}(K)$ is homeomorphic to the $m$-fold cyclic branched cover of the trefoil. These $(2, m)$-torus knots are alternating, but have genus greater than one. Thus, we will examine the case where alternating knots have genus one.

Theorem 1.1. Let $K$ be a 3 -strand pretzel knot $P(2 a+1,2 b+1,2 c+1)$, where $a, b, c>0$. Then $\Sigma_{3}(K)$ is an $L$-space.

This immediately implies the following.

Corollary 1.2. Let $K$ be a genus one, alternating knot. Then $\Sigma_{3}(K)$ is an L-space.

Proof. Suppose that $K$ is a genus one, alternating knot. By [3, Lemma 3.1] (see also [14]), $K$ is either a 2-bridge knot or a 3 -strand pretzel knot $P(\ell, m, n)$ where $\ell, m, n$ have the same sign. For a genus one, 2-bridge knot, Peters [15] shows that $\Sigma_{3}(K)$ is an $L$-space. If $K=P(\ell, m, n)$, then $\ell, m, n$ are odd by [6]. Thus Theorem 1.1 gives the conclusion.

Hence, the rest of paper is devoted to prove Theorem 1.1. In Section 2, we describe a link $\mathcal{L}$ whose double branched cover is homeomorphic to $\Sigma_{3}(K)$ for $K=P(2 a+1,2 b+1,2 c+1)$. Then Theorem 1.1 immediately follows from Theorem 2.2, which claims that the link $\mathcal{L}$ is quasi-alternating. Section 3 describes how to calculate determinants of links through Goeritz matrices. In Section 4 , we first argue the case where $a=1$. Section 5 completes the proof of Theorem 2.2 by using an inductive argument. The last section contains some remarks.

The author would like to thank the referee for helpful comments.

\section{Quasi-alternating links}

Let $K$ be a pretzel knot $P(2 a+1,2 b+1,2 c+1)$ with $a, b, c>0$, as illustrated in Figure 1. Here, each rectangular box consists of vertically right-handed halftwists of indicated number. This knot has cyclic period two such that its axis is drawn as the horizontal line. By taking the quotient of this action, the images of $K$ and the axis give a link $k \cup A$ in Figure 2. The central two boxes consist of vertical twists, and the right box consists of horizontal twists. Note that each component of this link is unknotted. Moreover, it is not a hard task to see that two components are interchangeable by using the left diagram of Figure 2.

Proposition 2.1. Let $\mathcal{L}$ be the link obtained as the lift of $A$ in $\Sigma_{3}(k)$, which is the 3 -sphere. Then $\Sigma_{2}(\mathcal{L})$ is homeomorphic to $\Sigma_{3}(K)$.

Proof. Let $M$ be the $\mathbb{Z}_{3} \oplus \mathbb{Z}_{2}$ branched cover of $k \cup A$, corresponding to the map $H_{1}\left(S^{3}-k \cup A\right) \rightarrow \mathbb{Z}_{3} \oplus \mathbb{Z}_{2}$ sending positively oriented meridians of $k$ and $A$ to $(1,0)$ and $(0,1)$, respectively. Then $M$ is homeomorphic to $\Sigma_{2}(\mathcal{L})$ and $\Sigma_{3}(K)$. 


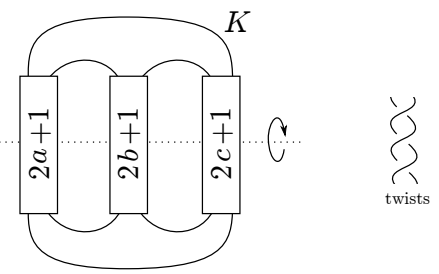

Figure 1. A pretzel knot $K=P(2 a+1,2 b+1,2 c+1)$ and twist convention.
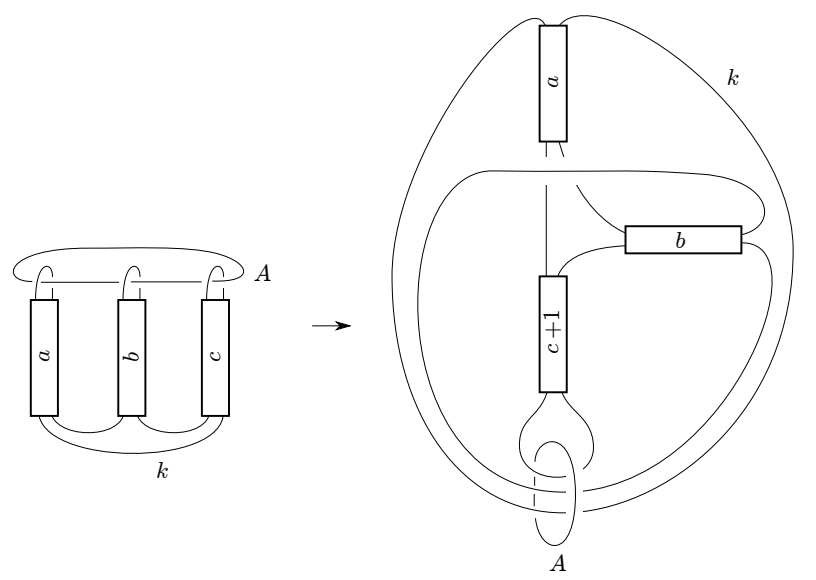

Figure 2. The link $k \cup A$.

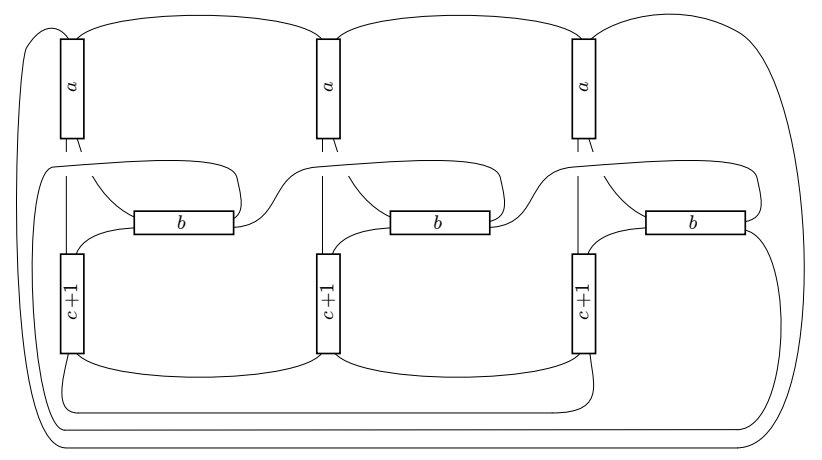

Figure 3 . The link $\mathcal{L}$.

This trick was found in $[11,16]$. After exchanging the position of $k$ and $A$ in Figure 2, we still have the same diagram (with exchanging the labels). Consider $\Sigma_{3}(k)$. Then the link $\mathcal{L}$ is as illustrated in Figure 3. 


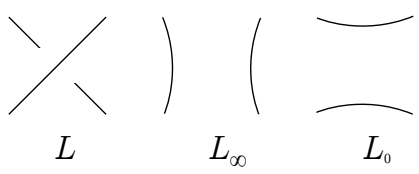

Figure 4. Resolutions.

We recall that the notion of quasi-alternating links [13]. The set of quasialternating links QA is the smallest set of links satisfying the following.

- The trivial knot belongs to QA.

- If a link $L$ has a diagram with crossing $c$ such that both of two links $L_{\infty}$ and $L_{0}$ obtained by smoothing $c$ as in Figure 4 belong to QA, and $\operatorname{det} L=\operatorname{det} L_{\infty}+\operatorname{det} L_{0}$, then $L$ belongs to QA.

As noted in Section 1, the double branched cover of a quasi-alternating link is an $L$-space, and any non-split alternating link is quasi-alternating (see [13]).

Theorem 2.2. The link $\mathcal{L}$ is quasi-alternating. Hence, $\Sigma_{2}(\mathcal{L})$ is an L-space.

The proof of this theorem is split into Sections 4 and 5 .

Proof of Theorem 1.1. By Proposition 2.1, $\Sigma_{3}(K)$ is homeomorphic to $\Sigma_{2}(\mathcal{L})$, which is an $L$-space by Theorem 2.2 .

\section{Determinant}

To show that the link $\mathcal{L}$ is quasi-alternating, it is necessary to calculate the determinant of $\mathcal{L}$ and those of various links arisen from $\mathcal{L}$ by resolutions. These calculations are done through Goeritz matrices (see [4]).

First, consider the checkerboard coloring of the diagram of $\mathcal{L}$ shown in Figure 3. The unbounded region is white, and this region will be ignored. The vertical $a$ right-handed half-twists at the upper left yield the white regions $\alpha_{1}, \alpha_{4}, \ldots, \alpha_{3 a-2}$ numbered from the top. Similarly, the white regions $\alpha_{2}, \alpha_{5}, \ldots, \alpha_{3 a-1}$ and $\alpha_{3}, \alpha_{6}, \ldots, \alpha_{3 a}$ appear at the upper center and the upper right. The three white regions just above horizontal $b$ twists are numbered $\alpha_{3 a+1}, \alpha_{3 a+2}, \alpha_{3 a+3}$ from the left. Finally, the white regions $\alpha_{3 a+4}, \alpha_{3 a+5}, \alpha_{3 a+6}$ are located on the left side of lower twists from the left. Figure 5 exhibits this numbering convention when $a=1$.

Figure 6 shows the convention of sign for each crossing. The $(3 a+6) \times(3 a+6)$ Goeritz matrix $G$ is defined as follows. For $i \neq j$, the $(i, j)$-entry of $G$ is the sum of signs at all the crossings between the regions $\alpha_{i}$ and $\alpha_{j}$. The $(i, i)$-entry is $-\sum \operatorname{sign}(c)$, where the sum is over all crossings $c$ around the region $\alpha_{i}$. Then it is well known that $|\operatorname{det} G|$ equals to the determinant of $\mathcal{L}$. 


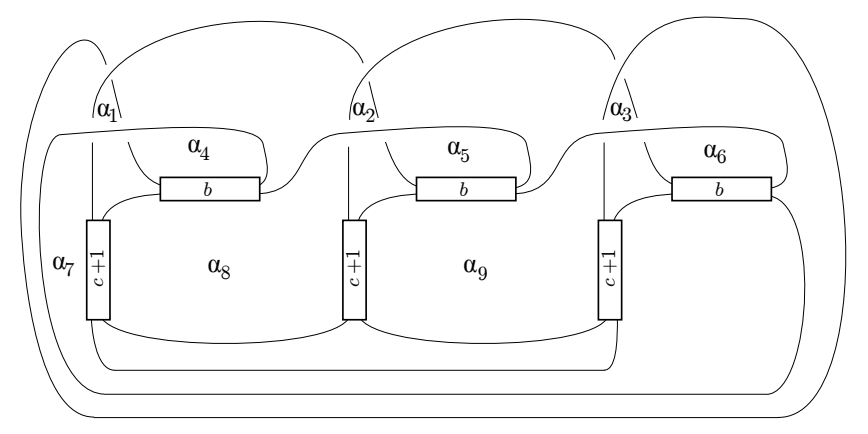

FiguRE 5. The link $\mathcal{L}$ and the white regions when $a=1$.

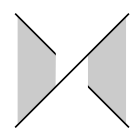

$+$

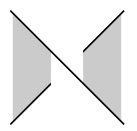

$-$

Figure 6 . Signs of crossing.

For example, if $a=1$, then the Goeritz matrix $G_{1}$ is

$\left(\begin{array}{c|ccc|ccc}-I & \multicolumn{2}{|c|}{-I} & \multicolumn{3}{c}{I} \\ \hline \multirow{2}{*}{-I} & \multicolumn{3}{|c|}{(b+1) I} & 0 & -b & 0 \\ & & & & -b & 0 & -b \\ \hline \multirow{2}{*}{I} & 0 & 0 & -b & b+2 c+1 & -c-1 & -c-1 \\ & -b & 0 & 0 & -c-1 & b+2 c+1 & -c-1 \\ & 0 & -b & 0 & -c-1 & -c-1 & b+2 c+1\end{array}\right)$,

where $I$ denotes the $3 \times 3$ identity matrix. Then a direct calculation shows $\operatorname{det} G_{1}=(3 b c+6 b+6 c+5)^{2}$. Since this value is positive, we have $\operatorname{det} \mathcal{L}=\operatorname{det} G_{1}$.

\section{The case where $a=1$}

The purpose of this section is to show that the link $\mathcal{L}$ is quasi-alternating when $a=1$. The link diagram $D$ is illustrated in Figure 5. For $i \in\{1,2,3\}$, let $c_{i}$ be the upper crossing of the white region $\alpha_{i}$. Let $\varepsilon_{i} \in\{*, \infty, 0\}$. We use the notation $L\left(\varepsilon_{1}, \varepsilon_{2}, \varepsilon_{3}\right)$ to express the link obtained from the link diagram $D$ by performing a resolution of type $\varepsilon_{i}$ at the crossing $c_{i}$. Here, if $\varepsilon_{i}=*$, then the crossing $c_{i}$ is not changed. If $\varepsilon_{i}=\infty$ or 0 , then $c_{i}$ is split vertically or horizontally, respectively, as in Figure 4.

Lemma 4.1. (1) $L(0,0, *)=L(0, \infty, 0)=P(b+c+1, b+c+1, b+c+1)$. Hence these are alternating.

(2) $L(0, \infty, \infty)=L(\infty, 0, \infty)=L(\infty, \infty, 0)$, and these are alternating. 


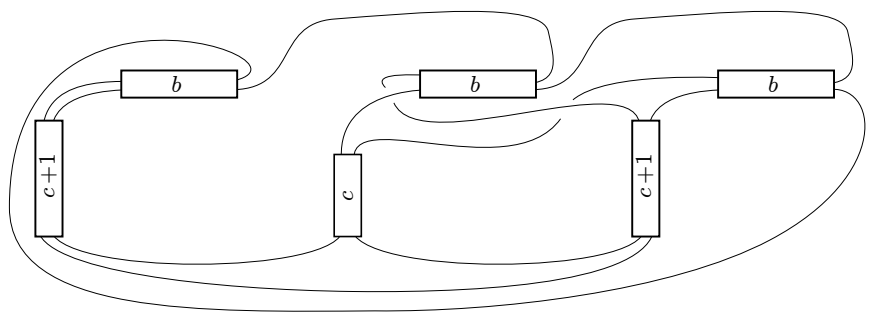

Figure $7 . L(0, \infty, \infty)$ is alternating.

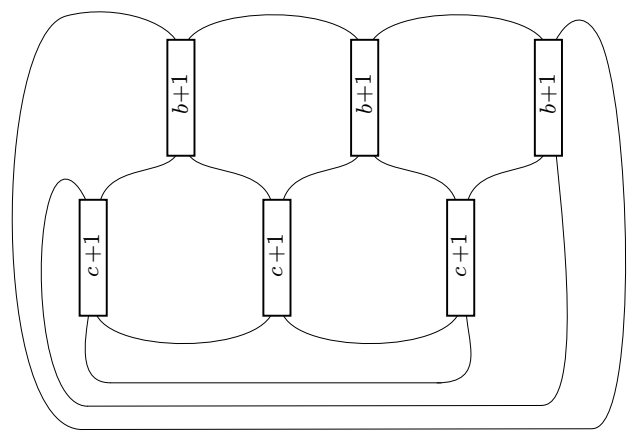

Figure 8. $L(\infty, \infty, \infty)$.

(3) $L(\infty, \infty, \infty)$ is quasi-alternating.

Proof. (1) This is obvious from their diagrams.

(2) The equivalences between three links follow from the symmetry. An alternating diagram of $L(0, \infty, \infty)$ is illustrated in Figure 7 .

(3) The link $L(\infty, \infty, \infty)$ is equivalent to one as in Figure 8. This link is shown to be quasi-alternating by Peters [15].

To conclude that $\mathcal{L}$ is quasi-alternating, we need the values of determinants of some of links $L\left(\varepsilon_{1}, \varepsilon_{2}, \varepsilon_{3}\right)$.

Recall that the diagram $D$ (Figure 5) of $\mathcal{L}$ yields the Goeritz matrix $G_{1}$ described in Section 3. For $L(0, *, *)$ (resp. $L(\infty, *, *)$ ), its diagram is obtained from $D$ by splitting the crossing $c_{1}$ horizontally (resp. vertically). Then, the corresponding Goeritz matrix is obtained from $G_{1}$ by replacing the $(1,1)$-entry with 0 , or deleting the first row and column, respectively. In this way, calculating determinants of the matrices gives Table 1 .

Theorem 4.2. Assume $a=1$. Then the link $\mathcal{L}$ is quasi-alternating. Furthermore, $L(0, *, *)$ and $L(\infty, *, *)$ are quasi-alternating.

Proof. By Lemma 4.1, $L(0, \infty, 0)$ and $L(0, \infty, \infty)$ are alternating. As shown in Table 1 , we have $\operatorname{det} L(0, \infty, *)=\operatorname{det} L(0, \infty, 0)+\operatorname{det} L(0, \infty, \infty)$. Hence 
TABLE 1. Determinants of links.

\begin{tabular}{c|c} 
Link & Determinant \\
\hline \hline$L(0, *, *)$ & $2(b+c+1)(3 b c+6 b+6 c+5)$ \\
$L(\infty, *, *)$ & $(3 b c+4 b+4 c+3)(3 b c+6 b+6 c+5)$ \\
\hline$L(0,0, *)$ & $3(b+c+1)^{2}$ \\
$L(0, \infty, *)$ & $(b+c+1)(6 b c+9 b+9 c+7)$ \\
\hline$L(\infty, 0, *)$ & $(b+c+1)(6 b c+9 b+9 c+7)$ \\
$L(\infty, \infty, *)$ & $(3 b c+3 b+3 c+2)(3 b c+5 b+5 c+4)$ \\
\hline$L(0, \infty, 0)$ & $3(b+c+1)^{2}$ \\
$L(0, \infty, \infty)$ & $2(b+c+1)(3 b c+3 b+3 c+2)$ \\
\hline$L(\infty, \infty, \infty)$ & $(3 b c+3 b+3 c+2)^{2}$
\end{tabular}

$L(0, \infty, *)$ is quasi-alternating. Similarly, because $L(0,0, *)$ is alternating and $\operatorname{det} L(0, *, *)=\operatorname{det} L(0,0, *)+\operatorname{det} L(0, \infty, *), L(0, *, *)$ is quasi-alternating. Also, we can verify that $L(\infty, *, *)$ is quasi-alternating by the same argument. Finally, the equation $\operatorname{det} \mathcal{L}=\operatorname{det} L(0, *, *)+\operatorname{det} L(\infty, *, *)$ implies the conclusion that $\mathcal{L}$ is quasi-alternating.

\section{Induction}

As in Section 4, we use the notation $L\left(a: \varepsilon_{1}, \varepsilon_{2}, \varepsilon_{3}\right)$ with $\varepsilon \in\{*, \infty, 0\}$ to denote the link obtained from $\mathcal{L}$ by performing the resolution of type $\varepsilon_{i}$ at the crossing $c_{i}$. Here, $c_{i}$ is located at the top of the white region $\alpha_{i}$. See Figure 3. Because we will use an inductive argument, the parameter $a$ is added. In particular, $\mathcal{L}=L(a: *, *, *)$.

Lemma 5.1. Suppose $a>1$.

(1) $L(a: 0,0, *)=L(a: 0, \infty, 0)=L(a: \infty, 0,0)=P(b+c+1, b+c+1, b+$ $c+1)$, and these are alternating.

(2) $L(a: 0, \infty, \infty)=L(a: \infty, 0, \infty)=L(a: \infty, \infty, 0)=L(a-1: 0, *, *)$.

(3) $L(a: \infty, \infty, \infty)=L(a-1: *, *, *)$.

Proof. These immediately follow from the diagrams.

Lemma 5.2. For $\mathcal{L}, L(a: 0, *, *)$ and $L(a: \infty, *, *)$,

$$
\begin{aligned}
\operatorname{det} \mathcal{L}= & (3 a b+3 b c+3 c a+3 a+3 b+3 c+2)^{2}, \\
\operatorname{det} L(a: 0, *, *)= & 2(b+c+1)(3 a b+3 b c+3 c a+3 a+3 b+3 c+2), \\
\operatorname{det} L(a: \infty, *, *)= & (3 a b+3 b c+3 c a+3 a+b+c) \\
& \cdot(3 a b+3 b c+3 c a+3 a+3 b+3 c+2) .
\end{aligned}
$$

Hence $\operatorname{det} \mathcal{L}=\operatorname{det} L(a: 0, *, *)+\operatorname{det} L(a: \infty, *, *)$. 
Proof. Let $G$ be the $(3 a+6) \times(3 a+6)$ Goeritz matrix obtained from the link diagram $D$ of $\mathcal{L}$. As in Section 3,

$$
G=\left(\begin{array}{cccc|ccc}
-2 I & I & & & & & \\
I & \ddots & & & & & \\
& & -2 I & I & & & \\
& & I & -2 I & I & O & O \\
\hline & & & I & & \text { G } \\
& & & O & & G 1
\end{array}\right),
$$

where $I$ is the $3 \times 3$ identity matrix, $O$ is the $3 \times 3$ zero matrix, and $G_{1}$ is exactly the $9 \times 9$ matrix given in Section 3 . To calculate its determinant, add the $i$-th column multiplied by $1 / 2$ to the $(i+3)$-th column for $i=1,2,3$. Then reduce the matrix to a $(3 a+3) \times(3 a+3)$ matrix. By repeating this process, we have $\operatorname{det} G=(-1)^{a-1} a^{3} \operatorname{det} G_{1}^{\prime}$, where $G_{1}^{\prime}$ is obtained from $G_{1}$ by replacing the upper left $3 \times 3$ block $-I$ with $-\frac{1}{a} I$. Thus $\operatorname{det} G=(-1)^{a-1}(3 a b+3 b c+$ $3 c a+3 a+3 b+3 c+2)^{2}$, and so $\operatorname{det} \mathcal{L} \stackrel{a}{=}(3 a b+3 b c+3 c a+3 a+3 b+3 c+2)^{2}$.

Consider the diagram of $L(a: 0, *, *)$ obtained from the diagram $D$ (Figure 3 ) by splitting the crossing $c_{1}$ horizontally. The corresponding Goeritz matrix $G_{0}$ is the above $G$ with replacing the $(1,1)$-entry with -1 . Add the first column to the 4 -th column, and the $i$-th column multiplied by $1 / 2$ to the $(i+3)$-th column for $i=2,3$. Then reduce the size as before. Repeating this gives $\operatorname{det} G_{0}=(-1)^{a-1} a^{2} \operatorname{det} G_{1}^{\prime \prime}$, where $G_{1}^{\prime \prime}$ is obtained from $G_{1}$ by replacing the $(i, i)$-entry with $0,-1 / a,-1 / a$, respectively, for $i=1,2,3$. Then we have $\operatorname{det} L(a: 0, *, *)=2(b+c+1)(3 a b+3 b c+3 c a+3 a+3 b+3 c+2)$.

Finally, the diagram of $L(a: \infty, *, *)$ is obtained from $D$ by splitting the crossing $c_{1}$ vertically. The corresponding Goeritz matrix $G_{\infty}$ is $G$ with deleting the first column and row. Add the $i$-th column multiplied by $1 / 2$ to the $(i+3)$ th column for $i=1,2$. Then reduce the size of matrix. Repeating this yields $\operatorname{det} G_{\infty}=(-1)^{a-3}(a-1) a^{2} \operatorname{det} G_{1}^{\prime \prime \prime}$, where $G_{1}^{\prime \prime \prime}$ is obtained from $G_{1}$ by replacing the $(i, i)$-entry with $-1 /(a-1),-1 / a,-1 / a$, respectively, for $i=1,2,3$. Then $\operatorname{det} L(a: \infty, *, *)=(3 a b+3 b c+3 c a+3 a+b+c)(3 a b+3 b c+3 c a+3 a+3 b+$ $3 c+2)$.

By a similar process to the proof of Lemma 5.2, we can calculate determinants of some other links, as in Table 2 . We omit the details.

TABLE 2. Determinants of links.

\begin{tabular}{c|c} 
Link & Determinant \\
\hline \hline$L(a: 0,0, *)$ & $3(b+c+1)^{2}$ \\
$L(a: 0, \infty, *)$ & $(b+c+1)(6 a b+6 b c+6 c a+6 a+3 b+3 c+1)$ \\
$L(a: \infty, \infty, *)$ & $(3 a b+3 b c+3 c a+3 a+2 b+2 c+1)(3 a b+3 b c+3 c a+3 a-1)$
\end{tabular}


Lemma 5.3. We have the following equations.

$$
\begin{aligned}
\operatorname{det} L(a: 0, *, *) & =\operatorname{det} L(a: 0,0, *)+\operatorname{det} L(a: 0, \infty, *), \\
\operatorname{det} L(a: 0, \infty, *) & =\operatorname{det} L(a: 0, \infty, 0)+\operatorname{det} L(a: 0, \infty, \infty), \\
\operatorname{det} L(a: \infty, *, *) & =\operatorname{det} L(a: \infty, 0, *)+\operatorname{det} L(a: \infty, \infty, *), \\
\operatorname{det} L(a: \infty, 0, *) & =\operatorname{det} L(a: \infty, 0,0)+\operatorname{det} L(a: \infty, 0, \infty), \\
\operatorname{det} L(a: \infty, \infty, *) & =\operatorname{det} L(a: \infty, \infty, 0)+\operatorname{det} L(a: \infty, \infty, \infty) .
\end{aligned}
$$

Proof. These immediately follow from Lemmas 5.1, 5.2 and Table 2.

Proof of Theorem 2.2. We prove both $L(a: 0, *, *)$ and $L(a: \infty, *, *)$ are quasialternating. Then, $\mathcal{L}=L(a: *, *, *)$ is quasi-alternating by Lemma 5.2 .

The proof is done by induction on $a$. By Theorem 4.2, the claim is true when $a=1$. Suppose $a>1$ and that the claim holds for $a-1$.

First, consider $L(a: 0, *, *)$. By the resolution at the crossing $c_{2}$, we obtain $L(a: 0,0, *)$ and $L(a: 0, \infty, *)$. For the latter, perform the resolution at the crossing $c_{3}$ to yield $L(a: 0, \infty, 0)$ and $L(a: 0, \infty, \infty)$. Then the claim that $L(a: 0, *, *)$ is quasi-alternating follows from the facts that $L(a: 0,0, *)$ and $L(a: 0, \infty, 0)$ are alternating (Lemma 5.1) and $L(a: 0, \infty, \infty)(=L(a-$ $1: 0, *, *))$ is quasi-alternating by our inductive assumption, coupled with the equations among determinants (Lemma 5.3). Similarly, we can show that $L(a: \infty, *, *)$ is quasi-alternating.

\section{Remarks}

(1) Boyer, Gordon and Watson [2] propose a conjecture that an irreducible rational homology 3 -sphere is an $L$-space if and only if its fundamental group is not left-orderable. For $K=P(2 a+1,2 b+1,2 c+1), \pi_{1} \Sigma_{2}(K)$ is not leftorderable, since $\Sigma_{2}(K)$ is a Seifert-fibered $L$-space ([2]). By Theorem 1.1, $\Sigma_{3}(K)$ is also an $L$-space. Hence it is expected that $\pi_{1} \Sigma_{3}(K)$ is not leftorderable. This is recently confirmed by [7].

(2) In Theorem 1.1, we showed that the 3-fold cyclic branched cover of any genus one, alternating pretzel knot is an $L$-space. Consider the genus one, non-alternating pretzel knot $P(-3,5,5)$. It is known that its double branched cover is not an $L$-space $([5,8])$. Thus we may not expect that its 3 -fold cyclic branched cover is an $L$-space.

(3) Let $K$ be a pretzel knot $P(3,3,-n)$ with $n \geq 3$, odd. If $n>3$, then $K$ is quasi-alternating, but $P(3,3,-3)$, which is $9_{46}$ in the knot table, is not quasi-alternating (see $[5,8])$. Nevertheless, $\Sigma_{2}(K)$ is always an $L$-space. By a similar argument to that of this paper, we can show that $\Sigma_{3}(K)$ is an $L$-space, but the details will be treated elsewhere.

(4) For genus one alternating pretzel knots $P(2 a+1,2 b+1,2 c+1)$, we may expect that the $d$-fold cyclic branched cover is an $L$-space for at least small $d \geq 4$. 


\section{References}

[1] J. Baldwin, Heegaard Floer homology and genus one, one-boundary component open books, J. Topol. 1 (2008), no. 4, 963-992.

[2] S. Boyer, C. McA. Gordon, and L. Watson, On L-spaces and left-orderable fundamental groups, Math. Ann. 356 (2013), no. 4, 1213-1245.

[3] S. Boyer and X. Zhang, Cyclic surgery and boundary slopes, Geometric topology (Athens, GA, 1993), 62-79, AMS/IP Stud. Adv. Math., 2.1, Amer. Math. Soc., Providence, RI, 1997.

[4] G. Burde and H. Zieschang, Knots, de Gruyter Studies in Mathematics, 5. Walter de Gruyter \& Co., Berlin, 2003.

[5] A. Champanerkar and I. Kofman, Twisting quasi-alternating links, Proc. Amer. Math. Soc. 137 (2009), no. 7, 2451-2458.

[6] D. Gabai, Genera of the arborescent links, Mem. Amer. Math. Soc. 59 (1986), no. 339, $1-98$.

[7] C. McA. Gordon and T. Lidman, Taut foliations, left-orderability, and cyclic branched covers, Acta Math. Vietnam. 39 (2014), no. 4, 599-635.

[8] J. Greene, Homologically thin, non-quasi-alternating links, Math. Res. Lett. 17 (2010), no. $1,39-49$.

[9] M. Hori, a private communication.

[10] $\mathrm{Y} . \mathrm{Hu}$, The left-orderability and the cyclic branched coverings, preprint, arXiv: 1311.3291.

[11] Y. Nakanishi, Primeness of links, Math. Sem. Notes Kobe Univ. 9 (1981), no. 2, 415-440.

[12] P. Ozsváth and Z. Szabó, On knot Floer homology and lens space surgeries, Topology 44 (2005), no. 6, 1281-1300.

[13] On the Heegaard Floer homology of branched double-covers, Adv. Math. 194 (2005), no. 1, 1-33.

[14] R. Patton, Incompressible punctured tori in the complements of alternating knots, Math. Ann. 301 (1995), no. 1, 1-22.

[15] T. Peters, On L-spaces and non left-orderable 3-manifold groups, preprint, arXiv: 0903.4495.

[16] M. Sakuma, Periods of composite links, Math. Sem. Notes Kobe Univ. 9 (1981), no. 2, 445-452.

[17] M. Teragaito, Fourfold cyclic branched covers of genus one two-bridge knots are Lspaces, Bol. Soc. Mat. Mex. 20 (2014), no. 2, 391-403.

[18] A. T. Tran, On left-orderability and cyclic branched coverings, preprint, arXiv: 1311.4262 .

Department of Mathematics and Mathematics Education

HiRoshima UNIVERSITY

1-1-1 KagamiYama, Higashi-Hiroshima, 739-8524, JAPAN

E-mail address: teragai@hiroshima-u.ac.jp 